\title{
Lean non-alcoholic fatty liver disease and development of diabetes: a cohort study
}

\author{
Dong Hyun Sinn',*, Danbee Kang2,*, Soo Jin $\mathrm{Cho}^{3}$, Seung Woon Paik1, Eliseo Guallar ${ }^{4,5}$, Juhee Cho ${ }^{2,4,5}$ and \\ Geum-Youn Gwak'
}

${ }^{1}$ Department of Medicine, Samsung Medical Center, Sungkyunkwan University School of Medicine, ${ }^{2}$ Department of Clinical Research Design and Evaluation, SAIHST, Sungkyunkwan University, ${ }^{3}$ Center for Health Promotion, Samsung Medical Center, ${ }^{4}$ Center for Clinical Epidemiology, Samsung Medical Center, Sungkyunkwan University, Seoul, South Korea, and ${ }^{5}$ Departments of Epidemiology and Medicine and Welch Center for Prevention, Epidemiology and Clinical Research, Johns Hopkins Medical Institutions, Baltimore, Maryland, USA

Correspondence should be addressed to G-Y Gwak or J Cho Email

gy.gwak@samsung.com or jcho@skku.edu

\section{Abstract}

Objective: Non-alcoholic fatty liver disease (NAFLD), a condition associated with multiple metabolic abnormalities, is frequently observed in normal weight individuals (lean NAFLD). The metabolic consequences of lean NAFLD, however, are not well characterized. Thus, this study aimed to evaluate the risk of incident diabetes in lean NAFLD.

Methods: This is a cohort study of 51,463 adults without diabetes, history of liver disease or cancer at baseline who participated in a regular health screening exam. Fatty liver was diagnosed by ultrasonography. The study outcome was the development of diabetes during follow-up.

Results: During 236,446.6 person-years of follow-up (median follow-up of 4.0 years), 5370 participants developed diabetes. In fully adjusted models, the hazard ratios (HRs) for incident diabetes comparing lean participants with NAFLD, overweight/obese participants without NAFLD and overweight/obese participants with NAFLD to lean participants without NAFLD, were 1.18 (95\% Cl: 1.03-1.35), 1.06 (0.98-1.14) and 1.45 (1.34-1.57), respectively. The fully adjusted HR for incident diabetes for lean NAFLD participants with low NAFLD fibrosis score (NFS) (<-1.455) and with intermediate-to-high NFS $(\geq-1.455)$ compared to lean participants without NAFLD were 1.32 (1.14-1.53) and 2.73 (2.10-3.55), respectively.

Conclusions: In this large cohort study, the presence and severity of NAFLD in normal weight adults was associated with an increased incidence of diabetes independently of established risk factors. Indeed, isolated lean NAFLD was a stronger risk factor for incident diabetes than the presence of overweight/obesity without NAFLD. Subjects with lean NAFLD require careful monitoring for the development of metabolic abnormalities.

\section{Introduction}

Non-alcoholic fatty liver disease (NAFLD), manifested by fat accumulation in the liver without secondary causes (1), is the most common liver disorder with an estimated prevalence of $25 \%$ worldwide (2). Obesity is the most common risk factor for NAFLD (3). Indeed, the classical phenotype of a NAFLD patient is an obese or overweight individual exhibiting some features of metabolic syndrome (1). However, between 8 and 19\% of cases of NAFLD occur in non-obese and non-overweight subjects (body mass index (BMI): $<25 \mathrm{mg} / \mathrm{kg}^{2}$ ), referred to as lean NAFLD $(4,5,6,7,8,9,10)$.

While lean NAFLD was associated with increased insulin resistance $(11,12)$, and with some metabolic abnormalities and cardiovascular risk factors (13), 
patients with lean NAFLD differ from overweight/obese NAFLD patients in genetic predisposition $(5,14)$, and severity of histological features (15). It is thus unclear if lean NAFLD has a different pathophysiology, phenotype characteristics and long-term outcomes compared to NAFLD in overweight/obese subjects. Specifically, data on the long-term association of lean NAFLD and incident diabetes is very limited $(16,17)$.

In this study, we assessed whether the presence and severity of NAFLD in lean individuals was associated with the future development of diabetes in a large cohort of asymptomatic adults without previous diabetes.

\section{Subjects and methods}

\section{Study population}

We conducted a retrospective cohort analysis of men and women of 18 years of age or older who underwent a comprehensive health screening exam at the Samsung Medical Center Health Promotion Center in Seoul, South Korea, from March 1st, 2003 to December 31st, 2013 (Fig. 1). Since our objective was to evaluate the longitudinal association between NAFLD and incidence of diabetes, the analysis was restricted to subjects who underwent at least two screening exams including determinations of fasting serum glucose and abdominal ultrasound (US) and who were free of diabetes at the first (baseline) exam $(n=63,484)$. We then excluded participants who had any of the following conditions at baseline: history of liver cirrhosis or positive hepatitis B surface antigen or hepatitis C virus antibodies $(n=3074)$; history of cancer $(n=1111)$; alcohol intake $\geq 30 \mathrm{~g} /$ day in men or $\geq 20 \mathrm{~g} /$ day in women at any point during follow-up $(n=5852)$; underweight (BMI

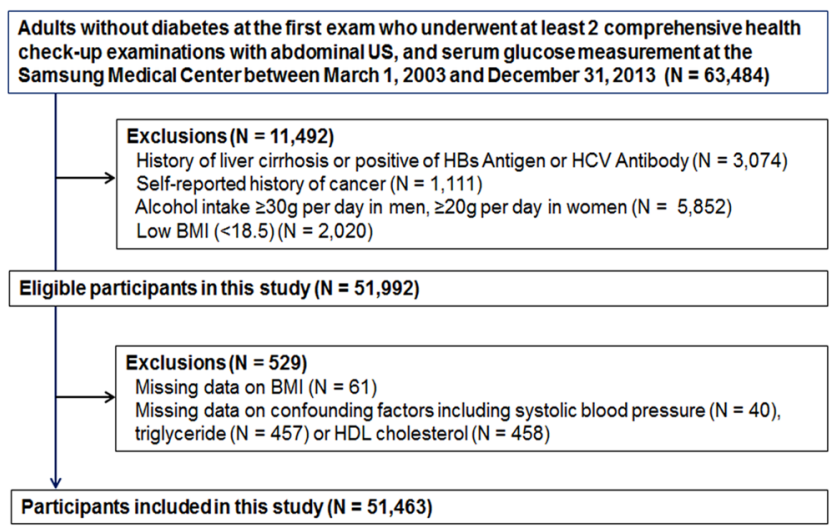

\section{Figure 1}

Flowchart of study participants $(n=51,931)$. $\left.<18.5 \mathrm{~kg} / \mathrm{m}^{2} ; n=2020\right)$. We further excluded participants with missing data on BMI $(n=61)$, and missing data on confounding factors; systolic blood pressure $(n=40)$, triglyceride $(n=457)$ or high-density lipoprotein (HDL) cholesterol $(n=458)$. Since study participants could have more than one exclusion criteria, the final sample size was 51,463 (26,804 men and 24,659 women).

The Institutional Review Board of the Samsung Medical Center approved this study and waived the requirement for informed consent as we used only de-identified data routinely collected during health screening visits.

\section{Data collection}

At each visit, demographic characteristics, smoking status, alcohol consumption, medical history and medication use were collected through standardized, self-administered questionnaires. Smoking status was categorized into never, former or current smoker. Current alcohol consumption was categorized into none or moderate $(<30 \mathrm{~g} /$ day in men and $<20 \mathrm{~g} /$ day in women).

Weight and height were measured by trained nurses. BMI was calculated as weight in kilograms divided by height in meters squared and was classified according to Asian-specific criteria (18) (underweight: $\mathrm{BMI}<18.5 \mathrm{~kg} / \mathrm{m}^{2}$; normal weight: BMI $18.5-22.9 \mathrm{~kg} / \mathrm{m}^{2}$; overweight: BMI of $23-24.9 \mathrm{~kg} / \mathrm{m}^{2}$ and obese: $\mathrm{BMI} \geq 25 \mathrm{~kg} / \mathrm{m}^{2}$ ).

Blood pressure was measured using a mercury sphygmomanometer after the subject had been seating for at least $10 \mathrm{~min}$. Hypertension was defined as a systolic blood pressure $\geq 140 \mathrm{~mm} \mathrm{Hg}$, a diastolic blood pressure $\geq 90 \mathrm{~mm}$ Hg or current use of antihypertensive medications. Fasting serum total cholesterol, triglycerides, HDL cholesterol and low-density lipoprotein (LDL) cholesterol levels were determined with an enzymatic colorimetric method. Hyperlipidemia was defined according to Adult Treatment Panel III criteria, as the presence of triglyceride levels $\geq 150 \mathrm{mg} / \mathrm{dL}$, HDL cholesterol levels $<40 \mathrm{mg} / \mathrm{dL}$ in men and $<50 \mathrm{mg} / \mathrm{dL}$ in women or use of lipid-lowering medications. Aspartate aminotransferase (AST), alanine aminotransferase (ALT) and gamma-glutamyltransferase levels were measured following the International Federation of Clinical Chemistry method.

Fasting serum glucose was measured by the hexokinase/glucose-6-phosphate dehydrogenase method. Diabetes was defined as the presence of fasting serum glucose levels $\geq 126 \mathrm{mg} / \mathrm{dL}$, a self-reported history of diabetes or current use of anti-diabetic medications. Hemoglobin A1c (HbA1c) was measured by highperformance liquid chromatography. As a sensitivity 
analysis, diabetes was alternatively defined as the presence of fasting serum glucose levels $\geq 126 \mathrm{mg} / \mathrm{dL}$, HbA1c levels $\geq 6.5 \%$, a self-reported history of diabetes or use of antidiabetic medications.

The Department of Laboratory Medicine and Genetics at Samsung Medical Center has participated in several proficiency testing programs operated by the Korean Association of Quality Assurance for Clinical Laboratory, the Asian Network of Clinical Laboratory Standardization and Harmonization, and the College of American Pathologists.

\section{Abdominal US}

Abdominal US imaging was performed using LogiQ E9 (GE Healthcare), iU22 xMatrix (Philips Medical Systems, Cleveland, OH, USA) or ACUSON Sequoia 512 equipment (Siemens, Issaquah, WA, USA) by experienced radiologists unaware of the study aims. Images were captured in a standard fashion with the patient in supine position with the right arm raised above the head. An US diagnosis of fatty liver was based on the following criteria: (1) hyperechogenic liver tissue with fine, tightly packed echoes; (2) reduction in posterior beam penetration; (3) discrepancy of echo amplitude between liver and kidney and (4) loss of echoes from the walls of the portal veins (19). Since we had already excluded participants with excessive alcohol use ( $\geq 30 \mathrm{~g} /$ day for men and $\geq 20 \mathrm{~g}$ /day for women) as well as those with other identifiable causes of fatty liver at baseline as described in the exclusion criteria, fatty liver was considered NAFLD.

Among individuals with NAFLD, we calculated the NAFLD fibrosis score (NFS) as $-1.675+0.037 \times$ age (years) $+0.094 \times$ BMI $\left(\mathrm{kg} / \mathrm{m}^{2}\right)+1.13 \times$ impaired fasting glucose/diabetes (yes $=1, \quad$ no $=0)+0.99 \times$ AST $/$ ALT ratio - $0.013 \times$ platelet count $\left(\times 10^{9} / \mathrm{l}\right)-0.66 \times$ albumin $(\mathrm{g} / \mathrm{dL})$. NFS was used to assess the severity of fibrosis (20) and to classify patients with NAFLD in two groups: highintermediate (NFS $\geq-1.455$ ) and low probability (NFS $<-1.455)$ of advanced fibrosis. The fibrosis-4 (FIB-4) score was calculated as age (years) $\times$ AST $(\mathrm{U} / \mathrm{L}) /$ [platelet count $\left.\left(10^{9} / \mathrm{L}\right) \times \operatorname{ALT}(\mathrm{U} / \mathrm{L})^{1 / 2}\right]$.

\section{Statistical analysis}

The study endpoint was the development of diabetes. Participants were followed from the baseline visit to the visit of diagnosis of diabetes or to the last available visit. Since incident diabetes occurred at an unknown time point between the visit of diagnosis and the previous visit (interval censoring), we used a flexible parametric proportional hazards model to evaluate the association between NAFLD status at baseline and incident diabetes. This model used restricted cubic splines in log time to estimate the hazard function and allowed for interval censored events.

We used three models with increasing degrees of adjustment to account for potential confounding factors at baseline. Model 1 was adjusted for baseline age, sex and year of visit. Model 2 was further adjusted for baseline smoking (never, former, current and missing) and alcohol intake (none, moderate and missing). Model 3 was further adjusted for baseline systolic blood pressure, fasting glucose, total cholesterol, HDL cholesterol, triglycerides ( $\log _{\mathrm{e}}$-transformed), use of antihypertensive medications and use of lipid-lowering medications. For sensitivity analyses, we repeated analyses using the alternative definition for diabetes incorporating HbA1c levels after excluding participants with missing data for HbA1c.

We also conducted analyses to evaluate impact of the severity of fibrosis in NAFLD on the incidence of diabetes. For NFS analyses, models were not adjusted for age or fasting glucose as these factors were included in the calculation of NFS. In addition, we performed stratified analyses to evaluate if the association of NAFLD with incident diabetes differed in pre-specified subgroups defined by age ( $<50, \geq 50$ years), sex, smoking (current, noncurrent), alcohol intake (none, moderate), hypertension (no, yes), hyperlipidemia (no, yes) and impaired fasting glucose (no, yes). All reported $P$ values were two-sided and the significance level was set at $P<0.05$. All analyses were performed using STATA version 14 (StataCorp LP).

\section{Results}

The mean (s.D.) age and BMI of study participants were 48.7 (9.1) years and $23.7(2.7) \mathrm{kg} / \mathrm{m}^{2}$ (BMI range 18.5-49.2 kg/ $\mathrm{m}^{2}$ ), respectively (Table 1). At baseline, 10.3 and $46.1 \%$ of study participants had lean NAFLD and overweight/ obese NAFLD, respectively. Among lean participants, those with NAFLD were more likely to be male, smokers, and metabolically unhealthy compared to those without NAFLD (Table 1).

During 236,446.6 person-years of follow-up (median follow-up 4.0 years), 5370 participants developed diabetes. Among lean participants, the 5-year cumulative incidences of diabetes were 5.5 and $11.0 \%$ in those without and with NAFLD, respectively. The 10-year cumulative incidence of diabetes in lean participants with 
Table 1 Baseline characteristics of the study participants by non-alcoholic fatty liver disease (NAFLD) and body mass index (BMI) status. Data are presented as mean (S.D.) or number (percentage).

\begin{tabular}{|c|c|c|c|c|c|}
\hline Characteristics & $\begin{array}{l}\text { Lean without NAFLD } \\
\qquad(n=19,722) \\
\end{array}$ & $\begin{array}{c}\text { Overweight/obese without } \\
\text { NAFLD }(n=15,899)\end{array}$ & $\begin{array}{l}\text { Lean with NAFLD } \\
\qquad(n=2262)\end{array}$ & $\begin{array}{l}\text { Overweight/obese with } \\
\text { NAFLD }(n=13,580)\end{array}$ & $P$ value \\
\hline Age, years & $46.9(9.0)$ & $49.7(9.1)$ & $50.8(8.7)$ & $50.2(8.8)$ & $<0.001$ \\
\hline Sex, men & 5904 (29.9) & $9383(59.0)$ & $1244(55.0)$ & $10,273(75.7)$ & $<0.001$ \\
\hline $\mathrm{BMI}, \mathrm{kg} / \mathrm{m}^{2}$ & $21.1(1.2)$ & $24.9(1.6)$ & $21.9(0.9)$ & $26.2(2.6)$ & $<0.001$ \\
\hline Smoking status & & & & & $<0.001$ \\
\hline Never & $13,856(70.3)$ & $8053(50.7)$ & $1188(52.5)$ & $5129(37.8)$ & \\
\hline Past & $909(4.6)$ & $1484(9.3)$ & $194(8.6)$ & $1780(13.1)$ & \\
\hline Current & $2268(11.5)$ & 2968 (18.7) & $470(20.8)$ & 3496 (25.7) & \\
\hline Missing & $2689(13.6)$ & $3394(21.4)$ & $410(18.1)$ & $3175(23.4)$ & \\
\hline Alcohol intake, g/day & & & & & $<0.001$ \\
\hline None & $8286(42.0)$ & $4666(29.4)$ & $817(36.1)$ & 3306 (24.3) & \\
\hline Moderate & $9582(48.6)$ & $9902(62.3)$ & $1258(55.6)$ & $9166(67.5)$ & \\
\hline Missing & $1854(9.4)$ & $1331(8.4)$ & $187(8.3)$ & $1108(8.2)$ & \\
\hline Hypertension & $2159(11.0)$ & $3680(23.2)$ & $498(22.0)$ & $4378(32.2)$ & $<0.001$ \\
\hline Systolic $\mathrm{BP}, \mathrm{mmHg}$ & $112.1(15.2)$ & $119.0(15.7)$ & $117.8(15.5)$ & $122.5(15.4)$ & $<0.001$ \\
\hline Use of AHM & $1077(5.5)$ & $2121(13.3)$ & $282(12.5)$ & $2576(19.0)$ & $<0.001$ \\
\hline Fasting glucose, $\mathrm{mg} / \mathrm{dL}$ & $88.0(8.6)$ & $91.1(9.1)$ & $92.6(9.5)$ & $94.9(10.2)$ & $<0.001$ \\
\hline $\mathrm{HbA} 1 \mathrm{c}, \%$ & $5.2(0.4)$ & $5.3(0.4)$ & $5.4(0.4)$ & $5.5(0.4)$ & $<0.001$ \\
\hline Hyperlipidemia & $4017(20.4)$ & $5558(30.0)$ & $1097(48.5)$ & $7940(58.5)$ & $<0.001$ \\
\hline Total cholesterol, mg/dL & $191.2(32.9)$ & $197.2(32.9)$ & $205.4(34.4)$ & $205.8(34.5)$ & $<0.001$ \\
\hline Triglycerides, mg/dL & $91.4(46.9)$ & $116.6(61.6)$ & $141.9(76.2)$ & $163.8(89.7)$ & $<0.001$ \\
\hline LDL cholesterol, mg/dL & $118.6(29.2)$ & $128.2(29.6)$ & $134.5(31.3)$ & $136.3(30.7)$ & $<0.001$ \\
\hline HDL cholesterol, mg/dL & $60.8(14.4)$ & $53.4(12.7)$ & $51.6(12.6)$ & $47.3(10.7)$ & $<0.001$ \\
\hline Use of LLM & $119(0.6)$ & $186(1.2)$ & 43 (1.9) & $244(1.8)$ & $<0.001$ \\
\hline$A L T, I U / L$ & $17.8(9.9)$ & $22.2(14.2)$ & $26.3(15.0)$ & $34.5(22.5)$ & $<0.001$ \\
\hline AST, IU/L & 20.9 (7.9) & $22.3(9.5)$ & $23.7(8.1)$ & $26.6(11.5)$ & $<0.001$ \\
\hline GGT, IU/L & $20.0(22.1)$ & $29.6(28.8)$ & $31.7(29.0)$ & $42.0(36.1)$ & \\
\hline
\end{tabular}

AHM, antihypertensive medications; ALT, alanine aminotransferase; AST, aspartate aminotransferase; BP, blood pressure; GGT, gamma-

glutamyltransferase; HDL, high-density lipoprotein; LDL, low-density lipoprotein; LLM, lipid-lowering medications; NAFLD, non-alcoholic fatty liver disease. Hemoglobin A1c $(n=494)$, ALT $(n=23)$, AST $(n=2)$ and GGT $(n=246)$ had missing data. For all other variables, the values were available for all participants.

NAFLD (14.1\%) was almost double compared to that in lean participants without NAFLD (27.3\%) (Supplementary Table 1 , see section on supplementary data given at the end of this article). In fully-adjusted models, the HRs comparing overweight/obese participants without NAFLD, lean NAFLD participants, and overweight/obese NAFLD participants to lean participants without NAFLD were 1.06 (95\% CI: 0.98-1.14), 1.18 (1.03-1.35) and 1.45 (1.34-1.57), respectively (Table 2 ). When diabetes was defined using the alternative definition including $\mathrm{HbA} 1 \mathrm{c}$, the corresponding HRs were 1.09 (1.01-1.18), 1.30 (1.14$1.47)$ and 1.55 (1.44-1.68), respectively (Supplementary Table 2).

When participants with lean NAFLD were stratified by NAFLD severity, the fully adjusted HR for incident diabetes in lean NAFLD participants with low NFS $(<-1.455)$ and with intermediate to high NFS ( $\geq-1.455)$ compared to lean participants without NAFLD were 1.32 (1.14-1.53) and 2.73 (2.10-3.55), respectively (Table 3). When overweight/obese participants with NAFLD were classified according to NFS, those with higher NFS also had an increased risk of incident diabetes (Table 3). When participants were stratified by another marker of NAFLD severity, FIB-4, lean NAFLD participants with higher FIB-4 score $(\geq 1.1)$ also showed increased risk of incident diabetes compared to those with lower FIB-4 score $(<1.1)$ (Supplementary Table 3).

The positive association between lean NAFLD and incident diabetes was observed in all subgroups analyzed (Fig. 2), although the association was stronger in those $<50$ compared to those $\geq 50$ years old ( $P$ value for interaction $=0.04$ ).

\section{Discussion}

In this large cohort study, we found that the presence NAFLD in lean participants was an independent risk factor for incident diabetes. The association between lean NAFLD and incident diabetes was stronger in participants with more advanced liver disease as indicated by a higher NFS or FIB-4 score. The positive association 
Table 2 Hazard ratios for incident diabetes by non-alcoholic fatty liver disease (NAFLD) and body mass index (BMI) status.

\begin{tabular}{|c|c|c|c|}
\hline & $\begin{array}{l}\text { No. of } \\
\text { subjects }\end{array}$ & $\begin{array}{l}\text { No. of } \\
\text { cases }\end{array}$ & $\begin{array}{c}\text { Incidence } \\
\text { rate* }\end{array}$ \\
\hline Lean without NAFLD & 19.722 & 1.241 & 13.8 \\
\hline Overweight/obese without NAFLD & 15.899 & 1.530 & 20.4 \\
\hline Lean with NAFLD & 2.262 & 278 & 27.0 \\
\hline $\begin{array}{l}\text { Overweight/obese with NAFLD } \\
P \text { value }\end{array}$ & 13.580 & 2.308 & 38.2 \\
\hline
\end{tabular}

\begin{tabular}{|c|c|c|}
\hline \multicolumn{3}{|c|}{ HR $(95 \% \mathrm{Cl})$} \\
\hline Model 1 & Model 2 & Model 3 \\
\hline Reference & Reference & Reference \\
\hline $1.29(1.20,1.40)$ & $1.29(1.20,1.39)$ & $1.06(0.98,1.14)$ \\
\hline $1.67(1.46,1.90)$ & $1.65(1.45,1.89)$ & $1.18(1.03,1.35)$ \\
\hline $2.34(2.18,2.52)$ & $2.33(2.16,2.50)$ & $1.45(1.34,1.57)$ \\
\hline$<0.001$ & $<0.001$ & $<0.001$ \\
\hline
\end{tabular}

$\mathrm{Cl}$, confidence interval; NAFLD, non-alcoholic fatty liver disease.

*Incidence rate per 1000 person-years.

Model 1 adjusted for age, sex and year of visit. Model 2 further adjusted for smoking (never, former, current and missing), and alcohol (none, moderate and missing). Model 3 further adjusted for systolic blood pressure, fasting glucose, total and HDL cholesterol, triglycerides (loge-transformed), use of antihypertensive medications and use of lipid-lowering medications

between NAFLD and incident diabetes was observed in all subgroups, including those with or without impaired fasting glucose, although it was strongest in lean NAFLD participants under 50 years old. Notably, participants with lean NAFLD had a higher risk of diabetes than overweight/ obese participants without NAFLD.

In a meta-analysis involving 19 observational studies with 296,439 individuals and nearly 16,000 cases of incident diabetes, individuals with NAFLD had a greater risk of incident diabetes than those without NAFLD (HR 2.22; 95\% CI 1.84-2.60) (21). However, there is limited data regarding the risk of incident diabetes among lean NAFLD individuals. A cohort study from Korea found a moderately strong hazard ratio (HR 2.69; 95\% CI, 1.72-4.20) comparing lean NAFLD patients to those without NAFLD, but this study included only 420 participants with lean NAFLD (16). Another cohort study from Japan $(n=4629)$ showed an increased risk of diabetes in non-overweight participants with NAFLD (HR 3.59; 95\% CI, 2.14-5.76), but they were not able to adjust metabolic risk factors such as blood pressure and cholesterol in multivariable analysis (17). To the best of our knowledge, our study is the largest cohort study that includes a comprehensive list of potential confounders and mediators to confirm the increased risk of diabetes among people with lean NAFLD compared to lean people without NAFLD.
In a meta-analysis of risk factors associated with NAFLD, the risk factor profile was similar in lean and overweight/obese NAFLD (13). Lean NAFLD patients also showed increased insulin resistance in the absence of other metabolic risk factors $(11,22)$. In the present study, lean NAFLD participants showed metabolically unhealthy profiles compared to lean participants without NAFLD. Furthermore, lean NAFLD participants were at higher risk of incident diabetes than overweight/obese participants without NAFLD. These findings suggest that NAFLD is an independent risk factor for metabolic abnormality irrespective of BMI status, and indicate that lean NAFLD individuals are at an increased risk of metabolic abnormalities. Lean NAFLD should be carefully monitored for the development of metabolic abnormalities and interventions that may decrease the risk of incident diabetes in lean NAFLD subjects should be designed and evaluated.

We categorized NAFLD according to the severity of fibrosis using the NFS, as histological data was lacking. Participants with more advanced NAFLD had a higher risk of incident diabetes. NAFLD encompassed a spectrum of disorders, from simple steatosis, to steatohepatitis and to advanced fibrosis (1). While hepatic insulin sensitivity was similar between patients with simple steatosis and with steatohepatitis, patients with steatohepatitis showed a more severe adipose tissue insulin resistance

Table 3 Hazard ratios for incident diabetes by severity of non-alcoholic fatty liver disease (NAFLD) and body mass index (BMI) status.

\begin{tabular}{|c|c|c|c|c|}
\hline & & HR $(95 \% \mathrm{Cl})$ & & \\
\hline & No NAFLD & NAFLD with NFS $<-1.455$ & NAFLD with NFS $\geq-1.455$ & $P$ values \\
\hline BMI categories & & & & $<0.001$ \\
\hline Lean & Reference & $1.32(1.14,1.53)$ & $2.73(2.10,3.55)$ & \\
\hline Overweight/obese & $1.19(1.10,1.29)$ & $1.56(1.43,1.70)$ & $2.95(2.67,3.27)$ & \\
\hline
\end{tabular}

$\mathrm{Cl}$, confidence interval; NAFLD, non-alcoholic fatty liver disease; NFS, NAFLD fibrosis score.

Adjusted for sex, year of visit, smoking (never, former, current and missing), alcohol (none, moderate and missing), systolic blood pressure, total and HDL cholesterol, triglycerides ( $\log _{\mathrm{e}}$-transformed), use of antihypertensive medications and use of lipid-lowering medications. For the NFS analyses, the models were not adjusted for age and fasting glucose as these factors are included in the calculation of the NFS. 

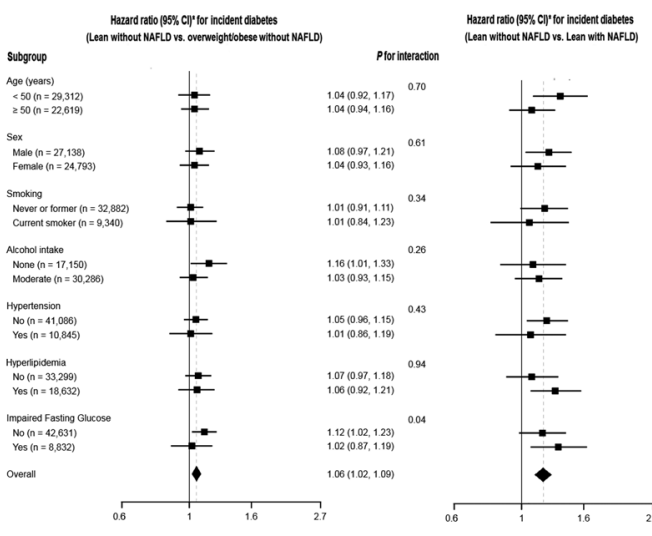

Figure 2

Hazard ratios for incident diabetes comparing (A) overweight/obese participants without non-alcoholic fatty liver disease (NAFLD), (B) lean NAFLD participants and $(C)$ overweight/obese NAFLD participants with lean participants without NAFLD in predefined subgroups at baseline. Adjusted for age, sex, year of visit, smoking (never, former, current and missing), alcohol (none, moderate and missing), systolic blood pressure, fasting glucose, total and HDL cholesterol, triglycerides ( $\log _{\mathrm{e}}$-transformed), use of antihypertensive medications and use of lipid-lowering medications. and a progressive reduction in whole-body insulin clearance compared to patients with simple steatosis (23). Steatohepatitis has been closely correlated with type 2 diabetes (24), which may explain a higher risk of diabetes in participants with advanced NAFLD.

In our analysis, the association between lean NAFLD and diabetes was stronger for younger participants. BMI was correlated better with adipose tissue compartments among younger ( $<50$ years of age) subjects compared with older subjects (21), which may explain a different degree of association in younger and older subjects. Yet, the exact reasons for these differences remain to be determined.

The prevalence of lean NAFLD varies according to ethnicity. Compared to Whites, the prevalence is lower in African Americans and higher in Hispanic Americans (25) and Asian (26). These differences may be related to genetic factors. A variant in the patatin-like phospholipase domain-containing 3 (PNPLA3) (rs738409[G], encoding I148M) is associated with increasing liver fat content, liver inflammation, fibrosis, and $\operatorname{NAFLD}(27,28)$. Genetic variation in the transmembrane 6 superfamily member 2 (TM6SF2) (rs58542926[T], encoding E167K) has been also linked to liver fat accumulation and increased risk of NASH $(29,30,31)$. In a study of 911 community subjects were randomly recruited in Hong Kong, non-obese NAFLD patients had a higher prevalence of the PNPLA3 rs738409[G] mutation compared to obese NAFLD patients (5). As we lacked genotypic data, we were unable to assess the impact of genetic variability on the association of lean NAFLD with incident diabetes.

Several other limitations also need to be considered in the interpretation of our findings. First, we classified lean and overweight/obese NAFLD based on BMI. The limitations of BMI as a marker of adiposity are well recognized (32), particularly as a marker of visceral adiposity, which plays a more important role than subcutaneous fat in the pathogenesis of insulin resistance (33). Second, we defined NAFLD by US after exclusion of secondary causes for steatosis. While US is widely used to diagnose fatty liver due to low cost, simplicity and safety, operator-dependency, reduced sensitivity and inability to quantify liver fat are major limitations of this test $(34,35,36)$. Third, NAFLD and BMI status change over time, but we did not reflect longitudinal changes in these characteristics over follow-up. Finally, our study population was comprised of asymptomatic Korean men and women who attended a comprehensive health screening exam. Hence, our findings need validation in other settings and in other race/ethnicity groups.

In summary, the presence and severity NAFLD in lean adults was associated with an increased incidence of diabetes independently of established risk factors. Indeed, isolated lean NAFLD was a stronger risk factor for the development of diabetes than the presence of overweight/ obesity without NAFLD. The positive association between lean NAFLD and incident diabetes increased with the severity of liver disease and was evident in all population subgroups evaluated, although it was stronger in younger participants. Our findings suggest that subjects with lean NAFLD require careful monitoring for the development of metabolic abnormalities. Future trials should explore whether preventive interventions can reduce the risk of diabetes in lean NAFLD patients. 


\section{Supplementary data}

This is linked to the online version of the paper at https://doi.org/10.1530/ EJE-19-0143.

\section{Declaration of interest}

The authors declare that there is no conflict of interest that could be perceived as prejudicing the impartiality of this study.

\section{Funding}

This research did not receive any specific grant from any funding agency in the public, commercial or not-for-profit sector.

\section{Author contribution statement}

Sinn DH and Gwak GY designed the study. Sinn DH, Kang D, Cho SJ and Paik SW collected the data. Kang D, Guallar E and Cho J performed the data analysis. Sinn DH, Kang D, Paik SW, Guallar E, Cho J and Gwak GY wrote the final report. All authors contributed to critical revision of the final report. Cho J and Gwak GY are guarantors. All the authors had full access to all of the data and can take responsibility for the integrity of the data and the accuracy of the data analysis.

\section{References}

1 Korean Association for the Study of the Liver (KASL). KASL clinical practice guidelines: management of nonalcoholic fatty liver disease. Clinical and Molecular Hepatology. 201319 325-348. (https://doi. org/10.3350/cmh.2013.19.4.325)

2 Younossi ZM, Koenig AB, Abdelatif D, Fazel Y, Henry L \& Wymer M. Global epidemiology of nonalcoholic fatty liver disease-Metaanalytic assessment of prevalence, incidence, and outcomes. Hepatology 201664 73-84. (https://doi.org/10.1002/hep.28431)

3 Chalasani N, Younossi Z, Lavine JE, Charlton M, Cusi K, Rinella M, Harrison SA, Brunt EM \& Sanyal AJ. The diagnosis and management of nonalcoholic fatty liver disease: practice guidance from the American Association for the Study of Liver Diseases. Hepatology 2018 67 328-357. (https://doi.org/10.1002/hep.29367)

4 Younossi ZM, Stepanova M, Negro F, Hallaji S, Younossi Y, Lam B \& Srishord M. Nonalcoholic fatty liver disease in lean individuals in the United States. Medicine 201291 319-327. (https://doi.org/10.1097/ MD.0b013e3182779d49)

5 Wei JL, Leung JC, Loong TC, Wong GL, Yeung DK, Chan RS, Chan HL, Chim AM, Woo J, Chu WC et al. Prevalence and severity of nonalcoholic fatty liver disease in non-obese patients: A population study using proton-magnetic resonance spectroscopy. American Journal of Gastroenterology 2015110 1306-1314; quiz 1315. (https:// doi.org/10.1038/ajg.2015.235)

6 Kim D \& Kim WR. Nonobese fatty liver disease. Clinical Gastroenterology and Hepatology 201715 474-485. (https://doi. org/10.1016/j.cgh.2016.08.028)

7 Nishioji K, Sumida Y, Kamaguchi M, Mochizuki N, Kobayashi M, Nishimura T, Yamaguchi K \& Itoh Y. Prevalence of and risk factors for non-alcoholic fatty liver disease in a non-obese Japanese population, 2011-2012. Journal of Gastroenterology 201550 95-108. (https://doi. org/10.1007/s00535-014-0948-9)

8 Fan JG, Kim SU \& Wong VW. New trends on obesity and NAFLD in Asia. Journal of Hepatology 201767 862-873. (https://doi. org/10.1016/j.jhep.2017.06.003)

9 Chalasani N, Younossi Z, Lavine JE, Diehl AM, Brunt EM, Cusi K, Charlton M \& Sanyal AJ. The diagnosis and management of nonalcoholic fatty liver disease: practice Guideline by the American
Association for the Study of Liver Diseases, American College of Gastroenterology, and the American Gastroenterological Association. Hepatology 201255 2005-2023. (https://doi.org/10.1002/hep.25762)

10 Fracanzani AL, Valenti L, Bugianesi E, Vanni E, Grieco A, Miele L, Consonni D, Fatta E, Lombardi R, Marchesini G et al. Risk of nonalcoholic steatohepatitis and fibrosis in patients with nonalcoholic fatty liver disease and low visceral adiposity. Journal of Hepatology 201154 1244-1249. (https://doi.org/10.1016/j. jhep.2010.09.037)

11 Sinn DH, Gwak GY, Park HN, Kim JE, Min YW, Kim KM, Kim YJ, Choi MS, Lee JH, Koh KC et al. Ultrasonographically detected non-alcoholic fatty liver disease is an independent predictor for identifying patients with insulin resistance in non-obese, nondiabetic middle-aged Asian adults. American Journal of Gastroenterology 2012107 561-567. (https://doi.org/10.1038/ajg.2011.400)

12 Gonzalez-Cantero J, Martin-Rodriguez JL, Gonzalez-Cantero A, Arrebola JP \& Gonzalez-Calvin JL. Insulin resistance in lean and overweight non-diabetic Caucasian adults: study of its relationship with liver triglyceride content, waist circumference and BMI. PLOS ONE 201813 e0192663. (https://doi.org/10.1371/journal. pone.0192663)

13 Sookoian S \& Pirola CJ. Systematic review with meta-analysis: risk factors for non-alcoholic fatty liver disease suggest a shared altered metabolic and cardiovascular profile between lean and obese patients. Alimentary Pharmacology and Therapeutics 201746 85-95 (https://doi.org/10.1111/apt.14112)

14 Sookoian S \& Pirola CJ. Genetic predisposition in nonalcoholic fatty liver disease. Clinical and Molecular Hepatology 201723 1-12. (https:// doi.org/10.3350/cmh.2016.0109)

15 Sookoian S \& Pirola CJ. Systematic review with meta-analysis: the significance of histological disease severity in lean patients with nonalcoholic fatty liver disease. Alimentary Pharmacology and Therapeutics 201847 16-25. (https://doi.org/10.1111/apt.14401)

16 Kim SS, Cho HJ, Kim HJ, Kang DR, Berry JR, Kim JH, Yang MJ, Lim SG, Kim S, Cheong JY et al. Nonalcoholic fatty liver disease as a sentinel marker for the development of diabetes mellitus in nonobese subjects. Digestive and Liver Disease 201850 370-377. (https:// doi.org/10.1016/j.dld.2017.12.018)

17 Fukuda T, Hamaguchi M, Kojima T, Hashimoto Y, Ohbora A, Kato T, Nakamura N \& Fukui M. The impact of non-alcoholic fatty liver disease on incident type 2 diabetes mellitus in non-overweight individuals. Liver International 201636 275-283. (https://doi. org/10.1111/liv.12912)

18 Kim MK, Lee WY, Kang JH, Kang JH, Kim BT, Kim SM, Kim EM, Suh SH, Shin HJ, Lee KR et al. 2014 Clinical practice guidelines for overweight and obesity in Korea. Endocrinology and Metabolism 2014 29 405-409. (https://doi.org/10.3803/EnM.2014.29.4.405)

19 Saverymuttu SH, Joseph AE \& Maxwell JD. Ultrasound scanning in the detection of hepatic fibrosis and steatosis. BMJ 1986292 13-15. (https://doi.org/10.1136/bmj.292.6512.13)

20 Angulo P, Hui JM, Marchesini G, Bugianesi E, George J, Farrell GC, Enders F, Saksena S, Burt AD, Bida JP et al. The NAFLD fibrosis score: a noninvasive system that identifies liver fibrosis in patients with NAFLD. Hepatology 200745 846-854. (https://doi.org/10.1002/ hep.21496)

21 Yim JY, Kim D, Lim SH, Park MJ, Choi SH, Lee CH, Kim SS \& Cho SH. Sagittal abdominal diameter is a strong anthropometric measure of visceral adipose tissue in the Asian general population. Diabetes Care 201033 2665-2670. (https://doi.org/10.2337/dc10-0606)

22 Kim HJ, Kim HJ, Lee KE, Kim DJ, Kim SK, Ahn CW, Lim SK, Kim KR, Lee HC, Huh KB et al. Metabolic significance of nonalcoholic fatty liver disease in nonobese, nondiabetic adults. Archives of Internal Medicine 2004164 2169-2175. (https://doi.org/10.1001/ archinte.164.19.2169)

23 Bril F, Lomonaco R, Orsak B, Ortiz-Lopez C, Webb A, Tio F, Hecht J \& Cusi K. Relationship between disease severity, hyperinsulinemia, 
and impaired insulin clearance in patients with nonalcoholic steatohepatitis. Hepatology 201459 2178-2187. (https://doi. org/10.1002/hep.26988)

24 Masarone M, Rosato V, Aglitti A, Bucci T, Caruso R, Salvatore T, Sasso FC, Tripodi MF \& Persico M. Liver biopsy in type 2 diabetes mellitus: steatohepatitis represents the sole feature of liver damage. PLOS ONE 201712 e0178473. (https://doi.org/10.1371/journal. pone.0178473)

25 Foster T, Anania FA, Li D, Katz R \& Budoff M. The prevalence and clinical correlates of nonalcoholic fatty liver disease (NAFLD) in African Americans: the multiethnic study of atherosclerosis (MESA). Digestive Diseases and Sciences 201358 2392-2398. (https://doi. org/10.1007/s10620-013-2652-7)

26 Kwon YM, Oh SW, Hwang SS, Lee C, Kwon H \& Chung GE. Association of nonalcoholic fatty liver disease with components of metabolic syndrome according to body mass index in Korean adults. American Journal of Gastroenterology 2012107 1852-1858. (https:// doi.org/10.1038/ajg.2012.314)

27 Romeo S, Kozlitina J, Xing C, Pertsemlidis A, Cox D, Pennacchio LA, Boerwinkle E, Cohen JC \& Hobbs HH. Genetic variation in PNPLA3 confers susceptibility to nonalcoholic fatty liver disease. Nature Genetics 200840 1461-1465. (https://doi.org/10.1038/ng.257)

28 Valenti L, Al-Serri A, Daly AK, Galmozzi E, Rametta R, Dongiovanni P, Nobili V, Mozzi E, Roviaro G, Vanni E et al. Homozygosity for the patatin-like phospholipase-3/adiponutrin I148M polymorphism influences liver fibrosis in patients with nonalcoholic fatty liver disease. Hepatology 201051 1209-1217. (https://doi.org/10.1002/ hep.23622)

29 Kozlitina J, Smagris E, Stender S, Nordestgaard BG, Zhou HH, Tybjaerg-Hansen A, Vogt TF, Hobbs HH \& Cohen JC. Exomewide association study identifies a TM6SF2 variant that confers susceptibility to nonalcoholic fatty liver disease. Nature Genetics 2014 46 352-356. (https://doi.org/10.1038/ng.2901)

30 Liu YL, Reeves HL, Burt AD, Tiniakos D, McPherson S, Leathart JB, Allison ME, Alexander GJ, Piguet AC, Anty R et al. TM6SF2 rs58542926 influences hepatic fibrosis progression in patients with non-alcoholic fatty liver disease. Nature Communications 201454309. (https://doi.org/10.1038/ncomms5309)

31 Koo BK, Joo SK, Kim D, Bae JM, Park JH, Kim JH \& Kim W. Additive effects of PNPLA3 and TM6SF2 on the histological severity of nonalcoholic fatty liver disease. Journal of Gastroenterology and Hepatology 201833 1277-1285. (https://doi.org/10.1111/jgh.14056)

32 Shea JL, Randell EW \& Sun G. The prevalence of metabolically healthy obese subjects defined by BMI and dual-energy X-ray absorptiometry. Obesity 201119 624-630. (https://doi.org/10.1038/ oby.2010.174)

33 McLaughlin T, Lamendola C, Liu A \& Abbasi F. Preferential fat deposition in subcutaneous versus visceral depots is associated with insulin sensitivity. Journal of Clinical Endocrinology and Metabolism 201196 E1756-E1760. (https://doi.org/10.1210/jc.2011-0615)

34 Hernaez R, Lazo M, Bonekamp S, Kamel I, Brancati FL, Guallar E \& Clark JM. Diagnostic accuracy and reliability of ultrasonography for the detection of fatty liver: a meta-analysis. Hepatology $20115 \mathbf{5 4}$ 1082-1090. (https://doi.org/10.1002/hep.24452)

35 Lee DH. Imaging evaluation of non-alcoholic fatty liver disease: focused on quantification. Clinical and Molecular Hepatology 201723 290-301. (https://doi.org/10.3350/cmh.2017.0042)

36 Bril F, Ortiz-Lopez C, Lomonaco R, Orsak B, Freckleton M, Chintapalli K, Hardies J, Lai S, Solano F, Tio F et al. Clinical value of liver ultrasound for the diagnosis of nonalcoholic fatty liver disease in overweight and obese patients. Liver International 201535 2139-2146. (https://doi.org/10.1111/liv.12840)

Received 28 February 2019

Revised version received 29 May 2019

Accepted 5 June 2019 